\title{
3D Printing of Intracranial Aneurysms Using Fused Deposition Modeling Offers Highly Accurate Replications
}

\author{
(D)A.M.J. Frölich, (D). Spallek, (D) L. Brehmer, (D).-H. Buhk, DD. Krause, (D). Fiehler, and (D) A. Kemmling
}

\begin{abstract}
BACKGROUND AND PURPOSE: As part of a multicenter cooperation (Aneurysm-Like Synthetic bodies for Testing Endovascular devices in 3D Reality) with focus on implementation of additive manufacturing in neuroradiologic practice, we systematically assessed the technical feasibility and accuracy of several additive manufacturing techniques. We evaluated the method of fused deposition modeling for the production of aneurysm models replicating patient-specific anatomy.
\end{abstract}

MATERIALS AND METHODS: 3D rotational angiographic data from 10 aneurysms were processed to obtain volumetric models suitable for fused deposition modeling. A hollow aneurysm model with connectors for silicone tubes was fabricated by using acrylonitrile butadiene styrene. Support material was dissolved, and surfaces were finished by using NanoSeal. The resulting models were filled with iodinated contrast media. 3D rotational angiography of the models was acquired, and aneurysm geometry was compared with the original patient data.

RESULTS: Reproduction of hollow aneurysm models was technically feasible in 8 of 10 cases, with aneurysm sizes ranging from 41 to 2928 $\mathrm{mm}^{3}$ (aneurysm diameter, 3-19 mm). A high level of anatomic accuracy was observed, with a mean Dice index of $93.6 \% \pm 2.4 \%$. Obstructions were encountered in vessel segments of $<1 \mathrm{~mm}$.

CONCLUSIONS: Fused deposition modeling is a promising technique, which allows rapid and precise replication of cerebral aneurysms. The porosity of the models can be overcome by surface finishing. Models produced with fused deposition modeling may serve as educational and research tools and could be used to individualize treatment planning.

ABBREVIATIONS: FDM = fused deposition modeling; ID = identification

E ndovascular aneurysm treatment is a continuously evolving field in which new treatment modalities are rapidly being developed and techniques are being refined, such as the recent introduction of intravascular flow diverters and intrasaccular flow disruptors. ${ }^{1,2}$ The safe and effective practice of endovascular neuroradiology demands an intimate understanding of the way devices work and interact with a given patient's vascular anatomy. In

Received May 8, 2015; accepted after revision June 17.

From the Department of Diagnostic and Interventional Neuroradiology (A.M.J.F., L.B., J.-H.B., J.F., A.K.), University Medical Center Hamburg-Eppendorf, Hamburg, Germany; and Technical University Hamburg-Harburg (D.K., J.S.), Institute of Product Development and Mechanical Engineering Design, Hamburg, Germany.

This work was supported by a grant from the Forschungszentrum Medizintechnik Hamburg.

Preliminary data on 4 aneurysm models were previously presented at: Annual Meeting of the German Society for Neuroradiology, October 23-25, 2014; Cologne, Germany.

Please address correspondence to Andreas M.J. Frölich, MD, Department of Diagnostic and Interventional Radiology, University Medical Center Hamburg-Eppendorf, Martinistr 52, 20246 Hamburg, Germany; e-mail: a.froelich@gmail.com

http://dx.doi.org/10.3174/ajnr.A4486 this regard, vascular models provide an ideal environment to allow familiarization with a given device. ${ }^{3}$ In addition, specific treatment challenges can be created to assess the behavior of devices in predefined situations. To enhance the applicability of such models for dedicated research and open the field of patientindividualized treatment planning, we aimed to develop patientindividual, anatomically precise vascular models that can be accessed with clinical endovascular devices. Multiple previous investigations have described the production of vascular models based on silicone casts. ${ }^{4-8}$ As part of a multicenter cooperation (Aneurysm-Like Synthetic bodies for Testing Endovascular devices in 3D Reality), we systematically assessed the technical feasibility and accuracy of different additive manufacturing methods for this purpose. With the primary goal of increasing production speed and decreasing costs, we evaluated the method of fused deposition modeling (FDM) for the production of hollow aneurysm models. The FDM material extrusion technology is one of the most widely installed and least cost-intensive among several additive manufacturing techniques. ${ }^{9}$ Our goal was to assess the 
Aneurysm and model characteristics

\begin{tabular}{llccc}
\hline $\begin{array}{c}\text { Model } \\
\text { ID }\end{array}$ & $\begin{array}{c}\text { Aneurysm } \\
\text { Geometry } \\
\text { and Location }\end{array}$ & $\begin{array}{c}\text { Patient } \\
\text { Aneurysm } \\
\text { Volume } \\
\left(\mathbf{m m}^{\mathbf{3}} \mathbf{)}\right.\end{array}$ & $\begin{array}{c}\text { Model } \\
\text { Aneurysm } \\
\text { Volume } \\
\left(\mathbf{m m}^{\mathbf{3}} \mathbf{)}\right.\end{array}$ & $\begin{array}{c}\text { Dice } \\
\text { Index of } \\
\text { Similarity } \\
(\%)\end{array}$ \\
\hline A & Saccular, basilar tip & 68.0 & 69.8 & $94.6 \%$ \\
B & Saccular, ICA & 220.2 & 180.1 & $90.0 \%$ \\
C & Fusiform, ICA & 731.8 & 722.5 & $91.7 \%$ \\
D & Saccular, VA & 278.0 & 263.4 & $95.9 \%$ \\
E & Giant, ICA & 2927.3 & 2843.0 & $97.3 \%$ \\
F & Fusiform, VA & 62.9 & $\mathrm{NA}$ & $\mathrm{NA}$ \\
G & Saccular, ACA & 36.9 & 40.9 & $91.3 \%$ \\
H & Saccular, AICA & 23.9 & $\mathrm{NA}$ & $\mathrm{NA}$ \\
I & Saccular, ICA & 153.6 & 153.6 & $95.3 \%$ \\
J & Saccular, ACA & 145.5 & 160.0 & $92.5 \%$ \\
\hline
\end{tabular}

Note:-ACA indicates anterior cerebral artery; VA, vertebral artery; NA, not applicable.

principal technical feasibility of FDM aneurysm model production and determine the geometric accuracy of the model and its potential use for treatment simulations.

\section{MATERIALS AND METHODS Study Design}

In this institutional review board-approved, single-center, retrospective study with waived individual consent, 3D rotational angiographic data from 10 aneurysms were manually selected to represent common aneurysm configurations. We included saccular $(n=7)$, fusiform $(n=2)$, and giant $(n=1)$ aneurysms with different dimensions and neck configurations (Table).

\section{Image Acquisition}

All clinical 3D rotational angiography data were acquired by using an Allura Xper FD 20/20 angiography system (Philips Healthcare, Best, the Netherlands) with the following acquisition parameters: 5 -second-rotational acquisition, $220^{\circ}$ rotation, 150 single frames at a frame rate of 30/s, 15- to 48-cm-detector FOV, 512 acquisition matrix. Images were reconstructed by using a soft-tissue kernel with an isotropic voxel size (edge length range, $0.1-0.3 \mathrm{~mm}$ ).

\section{Model Fabrication}

After patient selection, all image data were anonymized. 3D rotational angiographic data were processed by using Analyze software, Version 11.0 (AnalyzeDirect, Overland Park, Kansas). Vessels were segmented semiautomatically with supervision by using a region-growing algorithm with seeds placed in main arteries proximal to the aneurysm. Redundant vascular structures were isolated and stripped to finally obtain binary images representing the aneurysm and a short segment of the adjacent parent vessel. Small vascular branches such as the anterior choroidal artery were digitally shortened or removed if not immediately relevant to the aneurysm access route, to improve the physical stability of the models. Binary images of segmented vessels were inverted to obtain a vessel lumen model and converted to volumetric surface files suitable for additive manufacturing. Connectors for silicone tubes were digitally attached with the software CATIA V5 (Dassault Systèmes SA, Vélizy-Villacoublay, France). Using a Designjet Color 3D printer (Hewlett-Packard Development Company, Palo Alto, California), we fabricated a hollow aneurysm model from acrylonitrile butadiene styrene with $254-\mu$ m-layer thickness. Support material was removed mechanically and dissolved by using the Designjet 3D Removal System (Hewlett-Packard Development Company). Surfaces needed to be finished to overcome the inherent porosity of the raw models. We used 2 different methods for surface finishing: 1) placing the models in an acetone vapor bath for 90-120 seconds, and 2) infiltrating the models in a water-based impregnation agent NanoSeal (JELN Imprägnierung $\mathrm{GmbH}$, Schwalmstadt, Germany) for 15 minutes. The final models used for analysis were finished by using NanoSeal.

\section{Model Evaluation}

The resulting models were connected to silicone tubing and filled with iodinated contrast media (iopamidol, Iomeron 150; Bracco, Milan, Italy). 3D rotational angiography of the models was acquired on an Allura Xper FD 20 angiography system. Acquisition parameters were identical to the those in the clinical scan, with the exception of a fixed-detector FOV of $15 \mathrm{~cm}$.

The resulting images were again transformed to binary images and then coregistered with the original patient images by using a semiautomatic, rigid transformation matrix in Analyze 11.0. The volume of overlapping voxels was calculated. Aneurysm volumes were obtained and compared by using a paired $t$ test. The Dice index, which relates the amount of overlap to the overall object size, was calculated as a measure of anatomic precision of the models as follows: $S=(2 \times O L) /(A+B)$, where $A$ and $B$ are the aneurysm volumes in the model and patient data, $O L$ is the volume of overlapping voxels, and $S$ is the index of similarity, which ranges from zero for no overlap to 1 for identical, completely overlapping geometries. In addition, we subjectively compared aneurysm and parent vessel configuration for any noticeable differences in geometry. Contrast material leakage through porous parts of the models was noted, if present. Statistical analyses were performed by using MedCalc for Windows, Version 13 (MedCalc Software, Mariakerke, Belgium).

\section{RESULTS}

\section{Aneurysm Characteristics}

Morphologic characteristics of all aneurysms and corresponding vascular models are summarized in the Table. Model fabrication typically took approximately 3-6 days: Image segmentation and preparation took approximately 1-2 hours, the printing process took $2-5$ hours, removal of support material required 1-3 days (depending on the geometric complexity of the model), and finally, 1-2 further days were required for infusion of NanoSeal and drying. Among the selected aneurysm geometries, models could be successfully produced in 8 of 10 cases. In the remaining 2 cases, vascular segments in the access path or distal to the aneurysm were occluded. Occlusion occurred only in vascular segments with a diameter of $<1 \mathrm{~mm}$. The corresponding aneurysms were a 2-mm AICA aneurysm, which was not completely patent after manufacturing, and a 4-mm aneurysm arising from a hypoplastic $\mathrm{V} 4$ segment of the vertebral artery, measuring $<1 \mathrm{~mm}$; this vessel segment was occluded.

After manufacturing and removal of support material, the models were permeable to water. Surface finishing with both acetone vapor and infiltration made the models impermeable. However, some leakage of contrast occurred at the tube connectors with some of the models finished by using acetone vapor due 
to loss of contour of the tube connectors during the finishing process. All models in the final analysis were finished by using NanoSeal infiltration.

\section{Anatomic Precision}

Aneurysms with volumes ranging from 40.9 to $2927.3 \mathrm{~mm}^{3}$ and maximum diameters ranging from 3 to $19 \mathrm{~mm}$ were successfully reproduced (Figs 1 and 2). Mean aneurysm volumes were not significantly different for patient data versus vascular models (570 and $540 \mathrm{~mm}^{3}, P=.2$ ). The mean Dice index was $93.6 \% \pm 2.4 \%$. In 1 model, a small bleb on the aneurysm surface was observed, related to an imperfection in the fabrication process and was similar in appearance to a small daughter aneurysm. No other significant anatomic discrepancies were observed. No contrast leakage of the model wall was observed in the models produced for the final analysis. Integration of the models into a vascular model with flow was easy, and models could be accessed with a microcatheter. Due to the surface properties, wire and catheter navigation within the models was somewhat impeded by a relatively high friction.

\section{DISCUSSION}

The current analysis shows that aneurysm models produced with FDM offer a high level of accuracy. The method is applicable over a wide range of aneurysm geometries and sizes. After surface finishing, the models are rendered impermeable to water and can be easily incorporated into vascular flow models; the incorporation is facilitated by the digital addition of tube connectors. The resulting setup can be used to familiarize operators with a specific aneurysm geometry or to study the behavior of vascular implants in a given anatomy. Vessel segments $<1 \mathrm{~mm}$ were not reliably reproduced or were obstructed; this problem may be related to the printer and building material used and the surface-finishing procedure. It may represent a limitation of the FDM technique for manufacturing very small vascular branches and aneurysms. Currently available printers offer layer thicknesses below the $254-\mu \mathrm{m}$ layer thickness used in our project, which may allow manufacturing of even small vascular branches that were not patent with our current approach, but the current lower range is approximately $100 \mu \mathrm{m}$ (eg, $127 \mu \mathrm{m}$ on the Fortus printer; Stratasys, Eden Prairie, Minnesota). This range may be a drawback of FDM compared with other manufacturing methods, such as stereolithography and material jetting, which offer spatial resolutions of approximately $25 \mu \mathrm{m}$. Another limitation of FDM is the relatively slow build speed due to the inertia of the plotting heads and the required frequent changes in direction of the plotting heads. ${ }^{10}$

Advantages of FDM include the wide availability and relatively low cost of printers and building materials. One of the most interesting roles for additive manufacturing in interventional neuroradiology is the idea of in-hospital, on-demand production of aneurysm models directly available to physicians involved in aneurysm treatment. Our data show that FDM is principally suitable for this purpose but needs to be further compared with other additive manufacturing techniques for aneurysm model production.

Previously described neurovascular models have commonly used silicone; these models are typically produced by using a dissolvable core and casting liquid silicone around it. ${ }^{3,5}$ For patient-

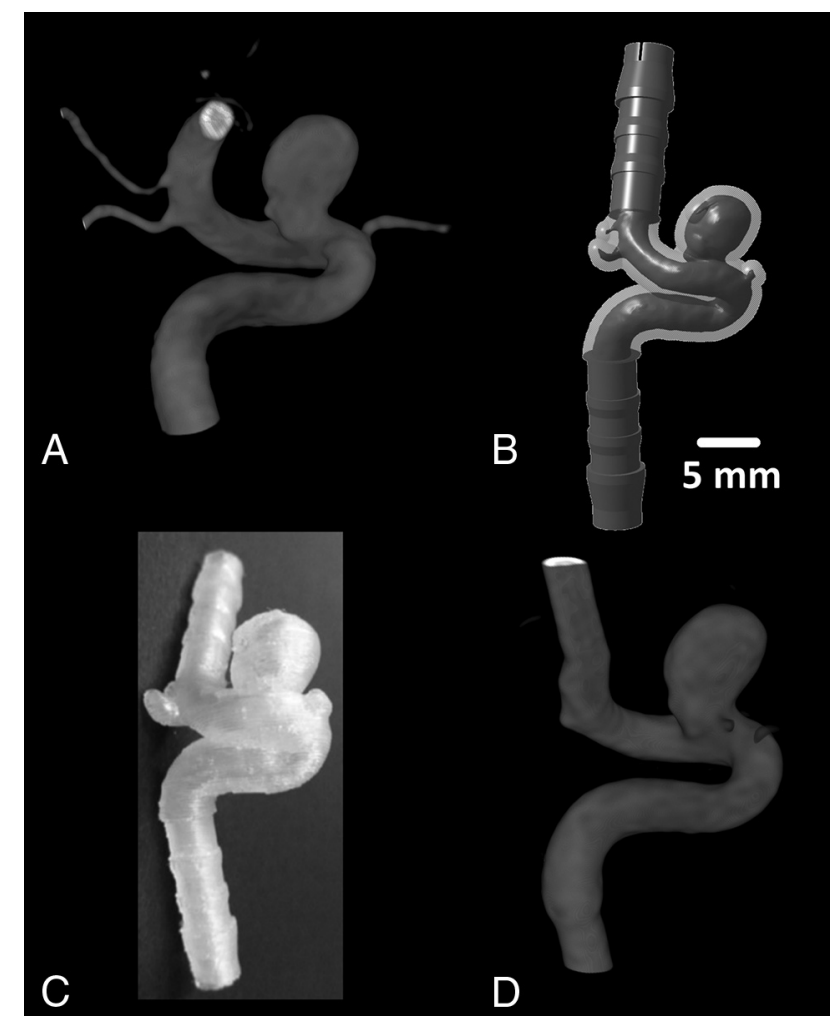

FIG 1. Model construction. In this case of a saccular supraophthalmic ICA aneurysm (model identification [ID] B), panels demonstrate 3D rotational angiography from the original patient data $(A)$, the digitized model with attached silicone tube connectors $(B)$, a photograph of the resulting FDM model $(C)$, and 3D rotational angiography obtained from this model $(D)$.

specific models, the core can be produced as a solid replica of the vessel lumen by direct additive manufacturing or by producing a mold that is then used to obtain wax replicas of the vessel lumen, which can be subsequently coated with silicone to produce the final model. ${ }^{5,7,8}$ This multistep manufacturing process has been reported to last approximately 2 weeks, ${ }^{4}$ whereas direct manufacturing of hollow models by using FDM and surface finishing in our study took approximately 3-6 days. If surface finishing could be avoided, possibly with new building materials, production speed may be further increased. The continuing development of additive manufacturing machines and technologies, such as printing a single object with different building materials, ${ }^{11}$ will further expand the tools available for producing vascular models and necessitates continuous evaluation of different printers and techniques.

Not all aneurysm geometries could be successfully produced. Particularly small vessel segments with an inner diameter of $<1$ $\mathrm{mm}$ were prone to short-segmental occlusions (Fig 3). Due to the layer-by-layer manufacturing process, vessel segments that run diagonally to the $\mathrm{x}-\mathrm{y}$ plane of the model are particularly susceptible to these occlusions, while those running parallel to the $\mathrm{z}$-axis should be less affected. Due to the complex geometry of the aneurysm models, occlusions can be difficult to predict. A different potential cause of interior occlusions or alteration of the interior surface is residue after surface finishing. Imperfections of the inner surface can impede wire and catheter movement and promote turbulent flow, which could affect hemodynamic measurements 


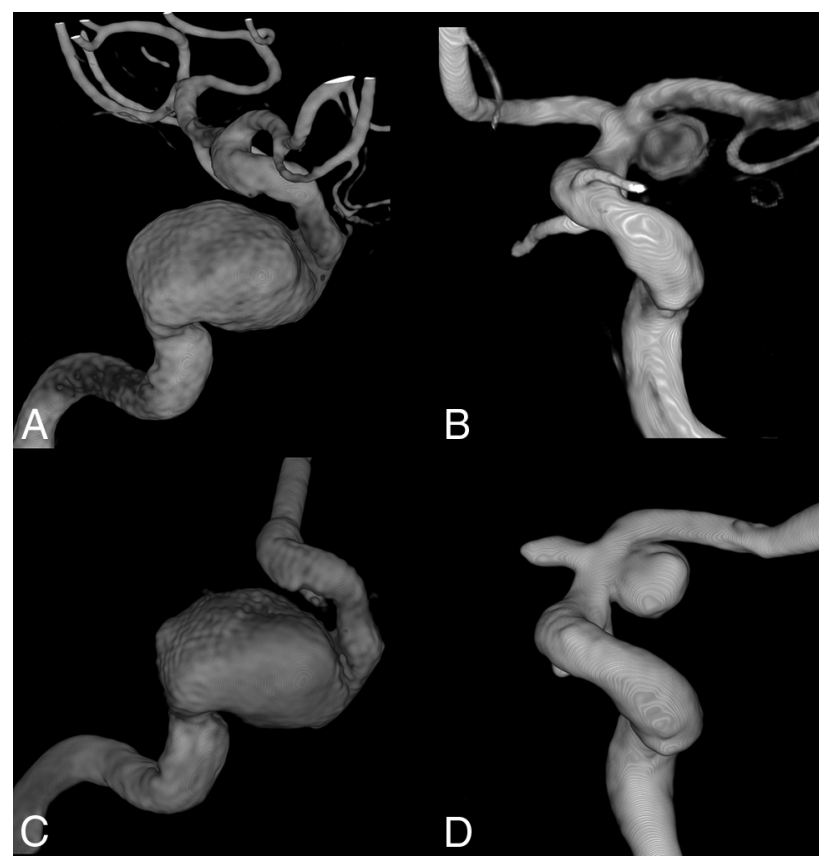

FIG 2. Sample aneurysm geometries. 3D rotational angiography demonstrates a giant fusiform ICA aneurysm (model ID E, left) and a supraophthalmic saccular ICA aneurysm (model ID A, right). Patient anatomy $(A$ and $B)$ and corresponding vascular models $(C$ and $D)$ are shown. Note that the anterior cerebral artery was purposely shortened in model ID A.



FIG 3. Small vascular branch occlusions. Multiplanar reformation from nonenhanced 3D rotational angiography of model ID F $(A)$ shows a short-segment occlusion (arrow) of the lumen. A similar, somewhat longer occlusion was encountered in model ID H (arrow in B).

performed in the models compared with the real endothelial surface.

Another limitation of the current models is the use of a semitranslucent building material. This currently restricts their use to an environment with fluoroscopic capabilities because catheter movement can barely be directly observed through the material; thus, the use of camera equipment instead of fluoroscopy is not currently feasible. However, transparent building materials for FDM are available commercially, and their use for aneurysm models should be further assessed. Other additive manufacturing methods, such as stereolithography, may offer an improved transparency and higher spatial resolution compared with FDM due to differences in optical properties of the resulting models. ${ }^{10} \mathrm{An}$ other limitation of the presented approach is the rigidity of the models. This means they will not realistically respond to catheter or coil forces, limiting the degree of realism when simulating procedures such as coil embolization. A growing understanding of the forces that occur inside the aneurysm and catheters during endovascular procedures ${ }^{12}$ and the mechanical properties of the vessel wall ${ }^{5}$ will help define the required properties for producing elastic aneurysm models with 3D printing.

Several critical parts of neurointerventional procedures depend heavily on the elasticity of the anatomic structures, such as the dislocation of catheters or coils, rupture of the aneurysm, and movement of vessels in the access path. Accurate replication of these properties will greatly increase the degree of realism. Elastic building materials are available for different additive manufacturing techniques including FDM, and their properties should be assessed. A further limitation of our study is its retrospective design with manual selection of different aneurysm morphologies. However, this approach allowed us to assess the feasibility of the technique by using a relatively small sample.

The clinical goal for the development of vascular models is improvement of patient safety by helping physicians acquire and maintain the necessary interventional skills. Apart from using physical models, neurointerventional procedures can also be practiced by using commercially available virtual reality simulators, which generate angiography-like images in a computer simulation controlled by specialized catheters and wires. The advantages of this approach are its great flexibility, broad range of available procedures, and practically limitless repeatability. ${ }^{13}$ However, haptic feedback is limited, and the behavior of catheters, coils, and other implants is simulated; these features severely affect the degree of realism, particularly in critical situations such as dislocation of implants and catheters. Furthermore, each device has to be specifically integrated into the simulation; this step limits the available interventional tools. In this regard, physical vascular models offer the advantage of using real catheter materials with realistic haptic feedback, which allows detailed studies of catheter and implant behavior. To our knowledge, no study exists directly comparing the effects of training for neurointerventional procedures with virtual reality simulators versus vascular models. Further assessment of the way these training environments help develop and maintain procedural skills and evaluation of their impact on procedural duration and complications seem highly promising targets for improving patient safety.

Possibly the most exciting prospect for patient-individual models is the idea of individualized treatment planning. If it could be shown that coils and other implants behave similarly in a $3 \mathrm{D}$ model compared with reality, an entire neurointerventional procedure could be performed in vitro to help plan the actual operation. The principal feasibility of this technique has recently been demonstrated by using a patient-specific silicone model. ${ }^{4}$ This approach may offer exciting advances with regard to patient safety. For inclusion into clinical practice, the speed of production and the cost of the models will be highly relevant, which may argue in favor of additive manufacturing over silicone-based 
model manufacturing. Furthermore, the ability to repetitively "treat" an individual aneurysm model or treat several models of the same aneurysm in parallel with different therapeutic approaches could improve our understanding of the suitability of the plethora of currently available aneurysm treatment modalities and may help in standardizing indications. An increased use of vascular models may also reduce the need for using animals as part of neurointerventional training courses and for a variety of research endeavors.

\section{CONCLUSIONS}

Hollow aneurysm models manufactured by FDM offer a high level of anatomic accuracy and can be easily integrated into vascular flow models for training and research purposes. Further assessment of FDM and comparison with other additive manufacturing techniques seems promising, particularly regarding the use of elastic and transparent building materials and the behavior of the models during simulated procedures.

Disclosures: Andreas M.J. Frölich—RELATED: Forschungszentrum Medizintechnik,* Comments: The work was supported by a grant from the Forschungszentrum Medizintechnik Hamburg, Hamburg, Germany; UNRELATED: Payment for Lectures (including service on Speakers Bureaus): speaker's honoraria from Siemens, Forchheim, Germany. Jan-Hendrik Buhk-UNRELATED: Board Membership: Clinical Advisory Board, Codman Neurovascular. Jens Fiehler-RELATED: Grant: BMBF, Hansestadt Hamburg. * ${ }^{*}$ Money paid to the institution.

\section{REFERENCES}

1. Papagiannaki C, Spelle L, Januel AC, et al. WEB intrasaccular flow disruptor-prospective, multicenter experience in 83 patients with 85 aneurysms. AJNR Am J Neuroradiol 2014;35:2106-11 CrossRef Medline

2. Brinjikji W, Murad MH, Lanzino G, et al. Endovascular treatment of intracranial aneurysms with flow diverters: a meta-analysis. Stroke 2013;44:442-47 CrossRef Medline

3. Paramasivam S, Baltsavias G, Psatha E, et al. Silicone models as basic training and research aid in endovascular neurointervention: a single-center experience and review of the literature. Neurosurg Rev 2014;37:331-37; discussion 337 CrossRef Medline

4. Kono K, Shintani A, Okada $\mathrm{H}$, et al. Preoperative simulations of endovascular treatment for a cerebral aneurysm using a patientspecific vascular silicone model. Neurol Med Chir (Tokyo) 2013;53: 347-51 CrossRef Medline

5. Chueh JY, Wakhloo AK, Gounis MJ. Neurovascular modeling: small-batch manufacturing of silicone vascular replicas. AJNR Am J Neuroradiol 2009;30:1159-64 CrossRef Medline

6. Wetzel SG, Ohta M, Handa A, et al. From patient to model: stereolithographic modeling of the cerebral vasculature based on rotational angiography. AJNR Am J Neuroradiol 2005;26:1425-27 Medline

7. Suzuki Y, Fujitsuka M, Chaloupka JC. Simulation of endovascular neurointervention using silicone models: imaging and manipulation. Neurol Med Chir (Tokyo) 2005;45:567-72; discussion 572-73 CrossRef Medline

8. Knox K, Kerber CW, Singel SA, et al. Rapid prototyping to create vascular replicas from CT scan data: making tools to teach, rehearse, and choose treatment strategies. Catheter Cardiovasc Interv 2005;65:47-53 CrossRef Medline

9. Wohlers T. Wohlers Report 2014: Additive Manufacturing and 3D Printing State of the Industry-Annual Worldwide Progress Report. Fort Collins: Wohlers Associates; 2014

10. Gibson I, Rosen D, Stucker B. Additive Manufacturing Technologies: 3D Printing, Rapid Prototyping, and Direct Digital Manufacturing. New York: Springer; 2015

11. Wurm G, Lehner M, Tomancok B, et al. Cerebrovascular biomodeling for aneurysm surgery: simulation-based training by means of rapid prototyping technologies. Surg Innov 2011;18:294-306 CrossRef Medline

12. Lamano JB, Bushnell GG, Chen H, et al. Force characterization of intracranial endovascular embolization: coil type, microcatheter placement, and insertion rate. Neurosurgery 2014;75:707-15; discussion 715-16 CrossRef Medline

13. Ahmed K, Keeling AN, Fakhry M, et al. Role of virtual reality simulation in teaching and assessing technical skills in endovascular intervention. J Vasc Interv Radiol 2010;21:55-66 CrossRef Medline 